\title{
El tratamiento antihipertensivo podría aumentar el riesgo cardiovascular en pacientes con diabetes y presión arterial sistólica menor de $140 \mathrm{mmHg}$
}

Antihypertensive treatment could increase cardiovascular risk in diabetics with a systolic blood pressure is less than $140 \mathrm{mmHg}$

Brunström M y col. BMJ 2016;352:i717.

\section{Objetivos}

Determinar el efecto del tratamiento antihipertensivo sobre la mortalidad y la morbilidad cardiovascular en sujetos con diabetes, a diferentes niveles de presión arterial (PA).

\section{Fuentes de datos}

Se realizó una búsqueda en las bases de datos CENTRAL, MEDLINE, EMBASE y BIOSIS.

\section{Selección de estudios}

Para ser elegibles, los estudios debían tener un seguimiento de al menos 12 meses e incluir 100 o más individuos con diabetes mellitus. Los ensayos debían comparar cualquier antihipertensivo contra placebo, dos antihipertensivos cualquiera vs. uno, o cualquier meta de PA vs. otra. Se excluyeron estudios estrictamente comparativos, que evaluaran un agente vs. otro, así como estudios con intervenciones combinadas.

\section{Extracción de datos}

Los puntos finales de interés pre-especificados fueron: mortalidad por cualquier causa, mortalidad cardiovascular y no cardiovascular, infarto de miocardio, accidente cerebrovascular, insuficiencia cardiaca e insuficiencia renal terminal. Se evaluó el riesgo de sesgo utilizando la herramienta Cochrane para riesgo de sesgo para ensayos clínicos aleatorizados. Los dos autores del estudio extrajeron los datos y evaluaron el riesgo de sesgo en forma independiente, resolviendo las diferencias a través de la re-evaluación de los datos originales y la discusión. Se calcularon los riesgos relativos para cada punto final de cada ensayo clínico y se sintetizaron los resultados usando un modelo de meta-análisis de efectos aleatorios.

\section{Resultados Principales}

Se incluyeron en este meta-análisis 49 ensayos clínicos aleatorizados, con un total de 73.738 participantes. El tratamiento antihipertensivo redujo el riesgo de mortalidad por cualquier causa, mortalidad cardiovascular, infarto de miocardio, ACV e insuficiencia renal terminal, si la PA sistólica basal era mayor de $150 \mathrm{mmHg}$. Si la PA sistólica basal se encontraba entre 140 y $150 \mathrm{mmHg}$, el tratamiento adicional redujo el riesgo de mortalidad por cualquier causa, infarto de miocardio e insuficiencia cardiaca. Sin embargo, si la PA sistólica basal era menor de 140 $\mathrm{mmHg}$, el tratamiento antihipertensivo aumentó el riesgo de mortalidad cardiovascular, con una tendencia hacia el incremento de mortalidad por cualquier causa (Ver Tabla 1).

Tabla 1. Efecto del tratamiento antihipertensivo sobre el riesgo relativo de mortalidad y enfermedad cardiovascular.

\begin{tabular}{|c|c|c|c|c|c|c|}
\hline & \multicolumn{6}{|c|}{ RR (IC95\%) } \\
\hline & Mortalidad total & Mortalidad cardiovascular & IAM & ACV & IC & IRT \\
\hline$>150 \mathrm{mmHg}$ & $0,89(0,80$ a 0,99$)$ & $0,75(0,57$ a 0,99$)$ & $0,74(0,63$ a 0,87$)$ & $0,77(0,65$ a 0,91$)$ & $0,73(0,53$ a 1,01$)$ & $0,82(0,71$ a 0,94$)$ \\
\hline 140 a $150 \mathrm{mmHg}$ & $0,87(0,78$ a 0,98$)$ & $0,87(0,71$ a 1,05$)$ & $0,84(0,76$ a 0,93$)$ & $0,92(0,83$ a 1,01$)$ & $0,80(0,66$ a 0,97$)$ & $0,91(0,74$ a 1,12$)$ \\
\hline$<140 \mathrm{mmHg}$ & $1,05(0,95$ a 1,16$)$ & $1,15$ (1 a 1,32$)$ & $1,00(0,87$ a 1,15$)$ & $0,81(0,53$ a 1,22$)$ & $0,90(0,79$ a 1,02$)$ & $0,97(0,80$ a 1,17$)$ \\
\hline
\end{tabular}

$\mathrm{ACV}=$ accidente cerebrovascular; $\mid \mathrm{AM}=$ infarto agudo de miocardio; IC=insuficiencia cardiaca; IC95\%=intervalo de confianza del $95 \%$; IRT=insuficiencia renal terminal; PAS=presión arterial sistólica; $R R=$ riesgo relativo.

Conclusiones

El tratamiento antihipertensivo reduce el riesgo de mortalidad y morbilidad cardiovascular en individuos con diabetes mellitus y una PA sistólica mayor de $140 \mathrm{mmHg}$. Si la PA sistólica es menor de $140 \mathrm{mmHg}$, el tratamiento adicional se asocia con un incremento del riesgo de mortali- dad cardiovascular, sin observarse beneficio.

Fuente de financiamiento/conflicto de interés de los autores: El estudio fue financiado por el Consejo estatal de Vasterbotten. Los autores declaran que no existieron conflictos de interés.

\section{Comentario}

Durante mucho tiempo se ha debatido cuáles son las metas de PA que deberían perseguirse en diabéticos. Hasta hace algunos años, se proponía que éstas debían ser más estrictas que en la población general, por debajo de $130 / 80 \mathrm{mmHg}^{1}$. Posteriormente, esta recomendación fue revisada sobre la base de la escasa evidencia que la sustentaba, estableciéndose la recomendación actual de meta de PA menor de 140/85 a $90 \mathrm{mmHg}^{2}$. En el presente meta-análisis se retoma esta cuestión, y sus conclusiones parecerían apoyar las recomendaciones actuales. Sin embargo, la lectura crítica del trabajo debe advertirnos sobre ciertas cuestiones importantes: 1) este metaanálisis no es un estudio diseñado para establecer umbrales de PA en diabéticos, de hecho, la mayoría de los estudios incluidos compararon diferentes tratamientos, por lo que el efecto aquí observado podría ser en realidad reflejo de las diferencias entre las intervenciones evaluadas; 2) si bien desde el título del trabajo se refieren a diabéticos en general, no todos los pacientes incluidos tenían diabetes y dentro de los que sí tenían ese diagnóstico, todos eran tipo 2 , medicados con antihipertensivos, por lo que no pueden extrapolarse

los datos a otros pacientes con diabetes, como los tipo 1 ; 3 ) los estudios incluidos eran muy heterogéneos en cuanto al antecedente de enfermedad cardiovascular previa, y desconocemos el tiempo de evolución de la diabetes, así como la concomitancia de fenómenos hipotensivos asociados a disautonomía, como la hipotensión ortostática y la hipotensión postprandial, ambos ligados con mayor morbi-mortalidad cardiovascular ${ }^{3,4}$ y que pudieron haber influido en los resultados.

\section{Conclusiones del comentador.}

Si bien la evidencia actual no parece sustentar un tratamiento de la hipertensión con metas mucho más exigentes en pacientes con diabetes que en la población general, es fundamental evaluar cada caso en particular: no es lo mismo -y no responderán de igual manera al tratamiento- un paciente joven, con mucha expectativa de vida y buena tolerancia a la medicación, que un paciente anciano frágil con diabetes, con mucho tiempo de evolución de la enfermedad en el que probablemente coexistan fenómenos hipotensivos disautonómicos.

Jessica Barochiner [ Sección Hipertensión Arterial, Servicio de Clínica Médica del Hospital Italiano de Buenos Aires. jessica.barochiner@ hospitalitaliano.org.ar ] Barochiner J. El tratamiento antihipertensivo podría aumentar el riesgo cardiovascular en pacientes con diabetes y presión arterial sistólica menor de 140 mmhg. Evid Act Pract Ambul. 2017;20(1):21. Comentado de: Brunström M y col. Effect of antihypertensive treatment at different blood pressure levels in patients with diabetes mellitus: systematic review and meta-analyses. BMJ 2016; 352:i717. PMID: 26920333.

\section{Referencias bibliográficas}

1. Mancia G, y col. ESH-ESC Task Force on the Management of Arterial Hypertension. 2007 ESH-ESC Practice Guidelines for the Management of Arterial Hypertension: ESHESC Task Force on the Management of Arterial Hypertension. J Hypertens. 2007;25(9):1751-62.

2. Mancia G, y col. Task Force for the Management of Arterial Hypertension of the European Society of Hypertension and the European Society of Cardiology. 2013 ESH/ESC Practice Guidelines for the Management of Arterial Hypertension. Blood Press. 2014;23(1):3-16.

3. Ricci F, y col. Cardiovascular morbidity and mortality related to orthostatic hypotension: a meta-analysis of prospective observational studies. Eur Heart J. 2015;36(25):1609-17. 4. Fisher AA, y col. Postprandial hypotension predicts all-cause mortality in older, low-level care residents. J Am Geriatr Soc. 2005;53(8):1313-20. 